\title{
Las motivaciones, competencias y factores de éxito para el emprendimiento y su impacto en el desempeño empresarial. Un análisis en las MIPyMES en el estado de Querétaro, México
}

The impact of motivations, competencies and success factors for entrepreneurship on business performance. An analysis in MSMES in Queretaro State, Mexico

\author{
Carmen Berenice Ynzunza Cortés ${ }^{* 1}$, Juan Manuel Izar Landeta ${ }^{2}$ \\ ${ }^{1}$ Universidad Tecnológica de Querétaro, México \\ ${ }^{2}$ Universidad Autónoma de San Luis Potosí, México
}

Recibido el 29 de noviembre de 2018; aceptado el 1 de enero de 2020

Disponible en Internet el: 14 de febrero de 2020

\section{Resumen}

El emprendimiento es una actividad que está cobrado diariamente una mayor relevancia como una forma de resolver los problemas económicos y de empleo que enfrentan todos los países, principalmente aquellos en vías de desarrollo. Debido a esto, es que los gobiernos están asumiendo un papel más proactivo en el desarrollo de iniciativas y políticas de soporte a los emprendedores y de fomento al potencial emprendedor. Por lo que, el objetivo de esta investigación es determinar el impacto de las motivaciones, competencias y los factores de éxito para el emprendimiento en el desempeño empresarial, mediante una encuesta aplicada a 429 emprendedores de micro, pequeñas y medianas empresas (MiPymes) del Estado de Querétaro, utilizando un muestreo no probabilístico de conveniencia y la técnica de ecuaciones estructurales. Los resultados encontrados prueban la existencia de relaciones estadísticamente significativas entre las motivaciones, las competencias y los factores de éxito para el emprendimiento, y el desempeño empresarial, lo que representa una aportación teórica y empírica. Desde la práctica, el conocimiento de estos factores puede guiar a las instituciones educativas y gubernamentales a fortalecer

\footnotetext{
*Autor para correspondencia

Correo electrónico bynzunza@uteq.edu.mx (C.B. Ynzunza Cortés).

La revisión por pares es responsabilidad de la Universidad Nacional Autónoma de México. 
estos aspectos para que el emprendedor pueda identificar las oportunidades de negocio, minimizar los riesgos asociados al emprendimiento e incentivar la creación de nuevas empresas, así como elevar su tasa de éxito.

Código JEL: L26, L60

Palabras clave: Emprendimiento; Emprendedor; Éxito emprendedor

\begin{abstract}
Entrepreneurship is an activity that has become increasingly relevant as a mechanism for solving economic and employment problems faced by all countries, mainly developing countries. As a result, governments have taken a more proactive role in developing initiatives and policies to support entrepreneurs and to increase the entrepreneurial potential, in order to boost economic growth and job creation. Therefore, the main aim of this research is to determine the impact of motivations, competencies and success factors for entrepreneurship on business performance.

It's a quantitative research of 429 entrepreneurs of micro, small and medium size enterprises (MSMEs), using a non-probabilistic convenience sampling method and structural modelling technique for testing the relationships. The results provide evidence that there are statistically significant relationships between motivations, competencies and success factors for entrepreneurship and business performance, which represents a theoretical and empirical framework contribution. From a practical point of view, knowledge of these factors could guide educational institutions and policy makers to create and strengthen these factors that allow entrepreneurs to identify business opportunities, reduce the risk associated with new ventures, as well as for encouraging new business creation and increasing its success rate.
\end{abstract}

JEL codes: L26, L60

Keywords: Entrepreneurship; Entrepreneur; Entrepreneurial success

\title{
Introducción
}

El emprendimiento es reconocido como uno de los principales impulsores del desarrollo de la economía de los países, por lo que los gobiernos han buscado a través del establecimiento de diversas acciones potencializar el crecimiento de la actividad emprendedora, como una alternativa de inserción laboral, que contribuya a solventar la demanda de empleo, mejorar la productividad, impulsar la innovación y por supuesto incidir en la economía. En los países desarrollados, el mayor número de empresas en funcionamiento corresponde a micro, pequeñas y medianas empresas, de no más de 100 empleados, las cuales han surgido como resultado de las oportunidades de mercado, las transformaciones tecnológicas, los cambios en las estructuras sociales y culturales; así también de las personas que anticipan los mismos y ven en el emprendimiento una oportunidad para mejorar sus ingresos económicos y su actividad laboral. 
En México, la contribución a la economía y desarrollo del país de este tipo de empresas es igualmente significativa, ya que representan el $99.8 \%$ de las entidades productivas, aportan el $52.0 \%$ del PIB y ocupan al $74.0 \%$ de la fuerza productiva total (INEGI, 2014). Sin embargo, se sabe que el $75.0 \%$ de los emprendimientos que se generan en el país fracasan y que la tasa de supervivencia es inferior a la de otros países del mundo como Brasil, Colombia y Estados Unidos (Failure Institute, 2016), por mencionar algunos. Entre las razones, por las que los emprendimientos no tienen el éxito esperado, se señalan además de la falta de recursos financieros, una deficiente operación y gestión; el desconocimiento del mercado y la escasa formación para emprender, debido a que la mayoría de los emprendedores, en este caso mexicanos, tienen poco conocimiento sobre cómo iniciar y operar un negocio (Secretaría de Economía, 2014) o simplemente no poseen los recursos, ya sea talento, conocimiento o herramientas para realizar esta actividad; ya que no fueron preparados para ello y más bien aprenden a partir del éxito o fracaso; y muchas veces emprenden porque provienen de familias que han tenido negocios antes.

Por otra parte, aunque existe información disponible en el país sobre emprendimiento, hay aspectos y relaciones que aún no han sido exploradas o los resultados no son concluyentes, por lo que se requieren más estudios que permitan profundizar en el conocimiento de los factores explicativos del éxito o fracaso de esta actividad. Aunado a ello, mucha de la información que existe sobre emprendimiento se ha realizado en empresas de países desarrollados o se ha enfocado a conocer las características de la personalidad de los emprendedores y las intenciones para emprender. Bajo este contexto, es clara la importancia de llevar a cabo este estudio sobre las motivaciones, competencias y factores de éxito para el emprendimiento y su impacto en el desempeño empresarial, buscando con ello aportar información para la toma de decisiones de inversión y la generación de acciones que fomenten el espíritu emprendedor e igualmente los aspectos que coadyuven a impulsar el número de emprendimientos y su tasa de éxito.

\section{El Emprendimiento en Querétaro}

Querétaro es un referente del desarrollo económico nacional, al registrar una tasa de crecimiento anual constante en la última década mayor a la tasa nacional promedio. En el Estado existen 92402 establecimientos. El $91.84 \%$ de estos son microempresas con menos de 10 empleados; el $6.28 \%$ son pequeñas empresas con entre 11 y 50 empleados. Mientras que, el $1.39 \%$ son de tamaño mediano con 51 a 250 empleados y tan solo el $0.49 \%$ son grandes empresas que emplean a más de 250 empleados. El aparato productivo está conformado principalmente por empresas del sector comercio y servicios no financieros con un $43.2 \%$ y $44.0 \%$ respectivamente. En contraste, con la industria manufacturera que representa el $8.7 \%$ y el $4.1 \%$ de otras actividades productivas (INEGI, 2019). Con relación al empleo, el 60.7\% 
de la población total ocupada del Estado labora para una MiPyme, lo que equivale a más de 480 mil personas (SEDESU, 2018). El sector de manufactura aporta el $35.79 \%$ del empleo y $70.2 \%$ de la producción bruta total, seguido del sector de servicios privados no financieros y comercio, $31.7 \%$ y $25.0 \%$ respectivamente (INEGI, 2014).

En cuanto a la actividad de emprendimiento, ésta representa un área de oportunidad para el Estado, por el nivel de desarrollo alcanzado, aunado a las características y potencial de la estructura empresarial existente y la tasa de crecimiento de la región, lo que genera un entorno favorable para iniciar una empresa, especialmente de tipo industrial y/o de base tecnológica, que cubra las necesidades de proveeduría y servicios que demanda la industria manufacturera. Con todo ello, según el reporte GEM (Global Entrepreneurship Monitor), el número de empresas nacientes, la tasa total de emprendimiento y de emprendimiento temprano exhiben tasas inferiores a la media nacional. Y se apertura mayormente empresas orientadas al consumidor, 89.3\% (Rehak et al., 2016), situación que contrasta con la vocación manufacturera del Estado.

\section{Revisión de literatura}

El emprendimiento representa una actividad compleja relacionada con la decisión de iniciar una empresa (Ventura y Quero, 2013), basada en una propuesta creativa de valor agregado que responde a una necesidad $\mathrm{u}$ área de oportunidad del entorno, en la que se asumen riesgos con la esperanza de obtener un beneficio económico o personal (Hisrich, Peters y Shepherd, 2013). En ella, se ponen a prueba las capacidades personales, de negocio y técnicas de la persona que emprende, a partir de su formación y experiencia (Léger y Kaloussis, 2006; Hisrich et al., 2013). Es a través de estas últimas, que los emprendedores obtienen el cúmulo de conocimientos, habilidades y aptitudes necesarias para emprender con éxito e igualmente son incentivados a desarrollar actividades asociadas con la creación de empresas (De Paco et al., 2011; Murali, Mohani y Yuzliani, 2009).

En la literatura sobre emprendimiento, son diversos los enfoques teóricos bajo los que se ha abordado su estudio: económico, conductual, sociológico, etc. En base a éstos, se argumenta que la decisión de emprender obedece mayormente a cuestiones económicas (Wennekers et al., 2005); a la posesión de determinadas características o rasgos psicológicos del emprendedor (Carsrud y Brännback, 2011) y a aspectos del entorno donde se emprende (Busenitz et al., 2003). Es así, que muchas investigaciones giran en torno a las razones que llevan a las personas a emprender y las condiciones que favorecen o limitan la iniciativa empresarial (Ventura y Quero, 2013; Olmos, González y Alcaraz, 2014; Canales, Román y Ovaldo, 2017; Marulanda y Morales, 2016). 


\section{Motivaciones para emprender}

Las motivaciones han sido tema de interés de muchos investigadores, quienes buscan conocer más sobre lo que impulsa a las personas a emprender, los problemas asociados al emprendimiento (Yalcin y Kapu (2008) y los factores de su éxito (Benzing, Chu y Kara, 2009). La motivación es reconocida como la fuerza necesaria para emprender, sortear las dificultades y uno de los más grandes predictores de los resultados y del éxito de la actividad emprendedora (Marulanda y Morales, 2016). En virtud de ello, han surgido distintas posturas que buscan conceptualizar y agrupar las motivaciones para emprender. Por ejemplo, Kuratko, Hornsby y Naffziger (1997), plantean la existencia de cuatro tipos de motivaciones: de recompensa extrínseca, intrínseca, autonomía y seguridad familiar. Yalcin y Kapu (2008), las agrupan en aspectos financieros, de reconocimiento, libertad y tradición familiar, mientras que otros las clasifican como "push" y "pull" factors. En las primeras, incluyen el desempleo, el crecimiento profesional y la insatisfacción laboral. Y como pull factors, están el deseo de independencia, de superación y las expectativas por alcanzar prestigio y beneficios económicos (Amit y Muller, 1995; Marulanda, Montoya y Vélez, 2014b). Bajo estas aproximaciones, se ha evidenciado que las motivaciones para iniciar una empresa están relacionadas principalmente con la búsqueda de un mejor ingreso, la seguridad en el empleo y la necesidad de trabajo y/o subsistencia (Benzing, Chu y Callanan, 2005; Benzing et al., 2009; Chu, Benzing y McGee, 2007). Así también, con el surgimiento de oportunidades y aspectos vinculados a la economía o el entorno (Coduras, Sainz y Ruiz, 2016; Carsrud y Brännback, 2011; Toca, 2010; Marulanda y Morales, 2016).

Igual, se han encontrado motivaciones relacionadas con las cuestiones de logro o reconocimiento (empresarial y personal) u otros factores propios del emprendedor, como el deseo de probar sus ideas y capacidades (Chu et al., 2007; Stefanovic, Prokic y Rankovic, 2010; Coduras et al., 2016); la búsqueda de desafíos (Swierczek y Hathai, 2011) o de satisfacción y crecimiento personal (Benzing et al., 2005). A la par, está el anhelo de ser su propio jefe y aspirar a un mejor estilo de vida (Cachon et al., 2013); provenir de una familia que ha emprendido antes y contar con relaciones personales y sociales en el ámbito empresarial; además de tener una fuerte vocación y atracción para los negocios (Coduras et al., 2016; Fuentelsaz et al., 2015).

Por otro lado, están las motivaciones que tienen que ver con el entrenamiento profesional y/o la falta de empleo. Siendo éstas referentes importantes en la intención y decisión de emprender, pues se ha encontrado que las personas tienen una mayor propensión a independizarse cuando la percepción sobre de sus capacidades es positiva y se ven a sí mismos como personas capaces de establecer una empresa; con un cierto potencial emprendedor (Quevedo, Izar y Romo, 2010) o cuando las experiencias laborales no son las deseadas (Dubini, 1988; 
Hamilton, 2000) y es evidente la falta de oportunidades de trabajo (Edelman, et al., 2016). Asimismo, cuando están dadas las circunstancias idóneas para emprender (Marulanda y Morales, 2016) y/o se tiene la influencia de familiares, amigos u otros, los cuales de alguna forma alientan o inhiben la decisión de independizarse e iniciar su propia empresa (Díaz, Hernández y Roldán, 2011; Moriano et al., 2012). La Tabla 1, resume las motivaciones para emprender encontradas en la literatura.

Tabla 1

Motivaciones para emprender

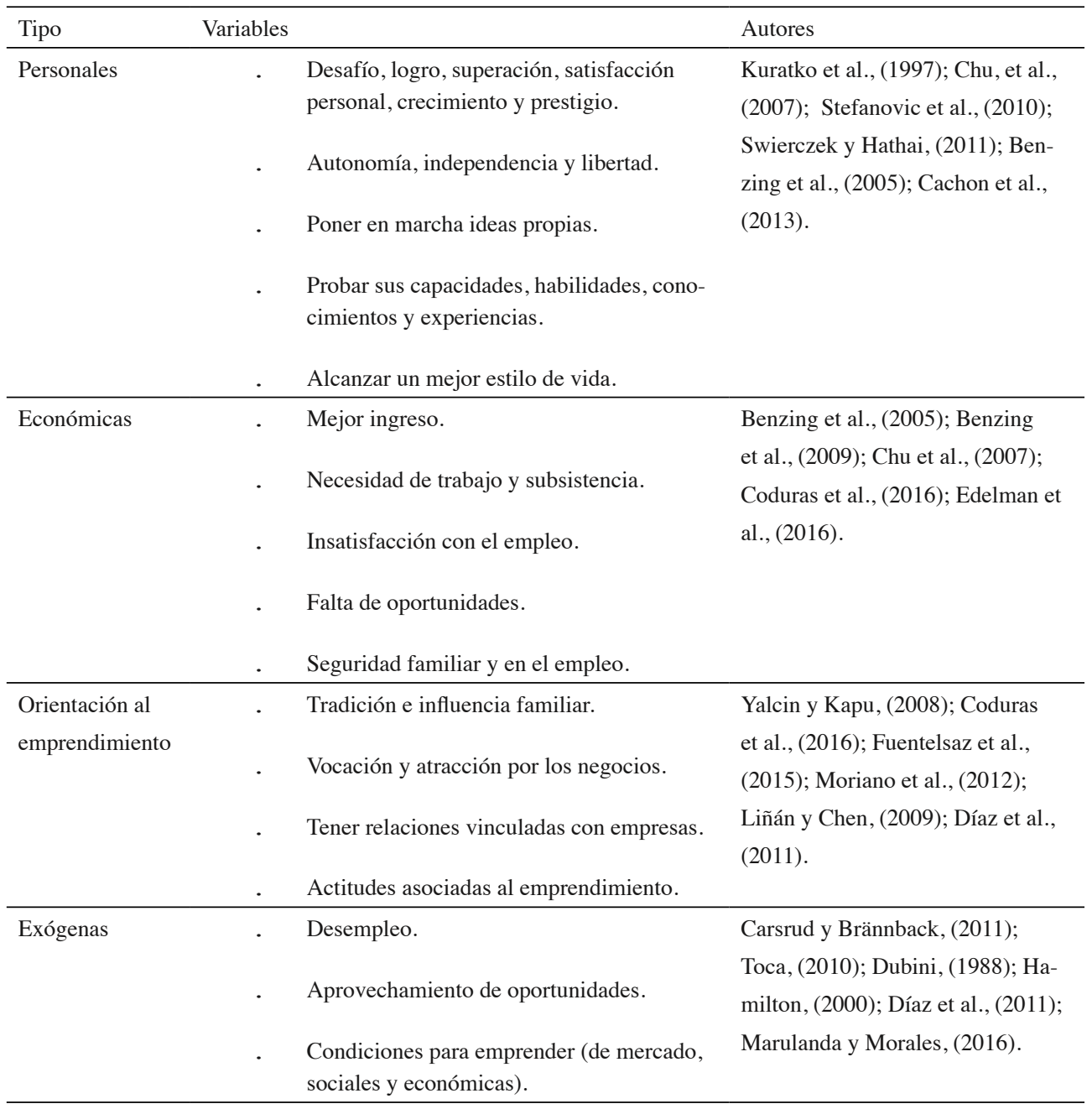

Fuente: Elaboración propia con base en la revisión de literatura. 


\section{Competencias para emprender}

Existen diversas conceptualizaciones sobre las competencias para emprender. Algunos investigadores las definen como todos aquellos conocimientos, habilidades, capacidades, destrezas, aptitudes, valores y actitudes con las que cuenta un emprendedor para llevar a cabo la tarea de iniciación de una empresa. En las mismas, están presentes diversos atributos individuales, sociales y adquiridos por el individuo a través de la educación formal y/o la experiencia (Gómez y Zatizábal, 2011; Olmos et al., 2014). En virtud de ello, es que estudios previos han enfatizado la importancia del perfil del emprendedor en el crecimiento empresarial (Colombo y Grilli, 2005) y han asociado determinados rasgos de la personalidad y cualidades del emprendedor con la predisposición de unas personas a emprender en relación a otras; entre ellas se mencionan: la determinación, el compromiso y la entrega total al éxito. También, el contar con iniciativa y capacidad para valorar las oportunidades (Muñoz, citado en Guerra, Hernández y Triviño, 2015). Además de, perseverancia y confianza en sí mismo (Amorós, Fernández y Tapia, 2011); ser innovador (Pablo, Santos y Bueno, 2004); tener cierta propensión al riesgo, capacidad para sobreponerse al fracaso y para establecer relaciones sociales; así también poseer habilidades de comunicación, liderazgo y negociación (Martínez y Carmona, 2009).

Por otra parte, está el currículo del emprendedor que parece ser otro de los factores de mayor influencia en la decisión de crear una empresa (Reynolds, 1991). A este respecto, hay quienes plantean que el éxito del emprendimiento depende de las aspiraciones y actividades del probable emprendedor (Acs y Szerb, 2011); también de sus expectativas, capacidades y de su potencial; el cual se ve fortalecido por sus conocimientos, habilidades gerenciales y experiencia empresarial (Ucbasaran et al., 2003). En este sentido, se argumenta que entre los factores más relevantes en la predicción y disminución de los riesgos de fracaso, sobresalen los aspectos de formación (Lee et al., 2006; Hmieleski, Corbett y Baron, 2013; Johansen, 2010; Minello, Alves y Da Costa, 2014); dado que la tasa de creación de nuevos negocios es mayor cuando se ha recibido educación sobre emprendimiento y cuando los emprendedores cuentan con experiencia y un buen nivel de competencia para innovar e introducir productos al mercado (Sarwoko, Surachman y Hadiwidjojo, 2013).

Asociado a ello, está el hecho de que las personas con mayores niveles de educación son también las más innovadoras, muestran una mayor tendencia a incursionar en nuevos modelos de negocio y son más proclives a incorporar en sus proyectos tecnologías novedosas (Pajarinen, Rouvinen y Ylä-Anttila, 2006); en virtud de que, la educación no sólo provee la formación técnica y gerencial para manejar negocios, sino también es el medio para generar las actitudes y comportamientos que se requieren para emprender e igual es un promotor del espíritu emprendedor (Castillo y Orlando, 2008) y el pensamiento creativo, al mismo tiempo que fortalece la autoestima y fomenta el emprendimiento (Consortium for Entrepreneurship 
Education, 2008). La Tabla 2, sintetiza las competencias para emprender identificadas en la literatura.

\section{Tabla 2}

Competencias para emprender

\begin{tabular}{|c|c|c|c|}
\hline Tipo & Variables & & Autores \\
\hline Capacidad & $\begin{array}{l}\cdot \\
\cdot \\
\cdot\end{array}$ & $\begin{array}{l}\text { Determinación y compromiso. } \\
\text { Iniciativa y visión. } \\
\text { Propensión al riesgo y capacidad para sobrepo- } \\
\text { nerse al fracaso. } \\
\text { Perseverancia y confianza en sí mismo. } \\
\text { Pasión e impulso. }\end{array}$ & $\begin{array}{l}\text { Muñoz, (2006) citado en Gue- } \\
\text { rra et al., (2015); Amorós et al., } \\
\text { (2011); Pablo, et al., (2004); Mar- } \\
\text { tínez y Carmona, (2009). }\end{array}$ \\
\hline $\begin{array}{l}\text { Habilidad y } \\
\text { aptitud }\end{array}$ & $\begin{array}{l}\cdot \\
\cdot \\
\cdot \\
\cdot\end{array}$ & $\begin{array}{l}\text { De liderazgo, comunicación y negociación. } \\
\text { Gerenciales. } \\
\text { Sociales y relacionales. } \\
\text { De innovación e incorporación de nuevas tecno- } \\
\text { logías y modelos de negocio. } \\
\text { Identificar y aprovechar oportunidades. }\end{array}$ & $\begin{array}{l}\text { Martínez y Carmona, (2009); } \\
\text { Ucbasaran et al., (2003); Pablo, } \\
\text { et al., (2004); Pajarinen et al., } \\
\text { (2006); Sarwoko et al., (2013). }\end{array}$ \\
\hline $\begin{array}{l}\text { Formación y } \\
\text { experiencia }\end{array}$ & $\cdot$ & $\begin{array}{l}\text { Profesional y empresarial. } \\
\text { Conocimiento del negocio. } \\
\text { Experiencia en negocios. } \\
\text { Redes de emprendedores. } \\
\text { Políticas, regulaciones, apoyos. } \\
\text { Condiciones del entorno. }\end{array}$ & $\begin{array}{l}\text { Lee et al., (2006); Sarwoko et al., } \\
\text { (2013); Hmieleski et al., (2013); } \\
\text { Johansen, (2010); Pablo, et al., } \\
\text { (2004); Ucbasaran et al., (2003); } \\
\text { Marulanda y Morales, (2016); } \\
\text { Gómez y Zatizábal, (2011); Mine- } \\
\text { llo et al., (2014); Kantis Angelelli } \\
\text { y Koenig, (2004); Elfring y Hul- } \\
\text { sink, (2007). }\end{array}$ \\
\hline
\end{tabular}

Fuente: Elaboración propia con base en la revisión de literatura. 


\section{Factores de éxito para el emprendimiento}

La evidencia empírica ha demostrado que en la experiencia emprendedora confluyen diversos elementos. Entre las variables estudiadas vinculadas a los emprendimientos no fallidos están el perfil del emprendedor; sus características personales; formación y experiencia previa en la iniciación y gestión de empresas (Chu et al., 2007; Stefanovic et al., 2010; Gonzalo, Federico y Kantis, 2013; Hamidi, Wennberg y Berglund, 2008). También, se refiere la estrategia de negocio de la cual dependen los factores críticos del éxito emprendedor relacionados con el servicio y la atención al cliente; el ofertar un buen producto a un precio competitivo; contar con una infraestructura de soporte sólida, así como estrategias de mercadotecnia y ventas efectivas (Stefanovic et al., 2010). A la par de trabajar duramente, mantenerse siempre bien informado, conocer el entorno y tener redes de contactos sociales y empresariales; además de otros aspectos estratégicos (Stefanovic et al., 2010; Chu, et al., 2007; Capelleras y Kantis, 2009) como acceso a recursos (financieros, humanos, de información) y estar integrado a una red de emprendedores (Gonzalo et al., 2013; Elfring y Hulsink, 2007), entre otros. La Tabla 3 , concentra los factores de éxito citados en investigaciones previas.

Tabla 3

Factores de éxito para el emprendimiento

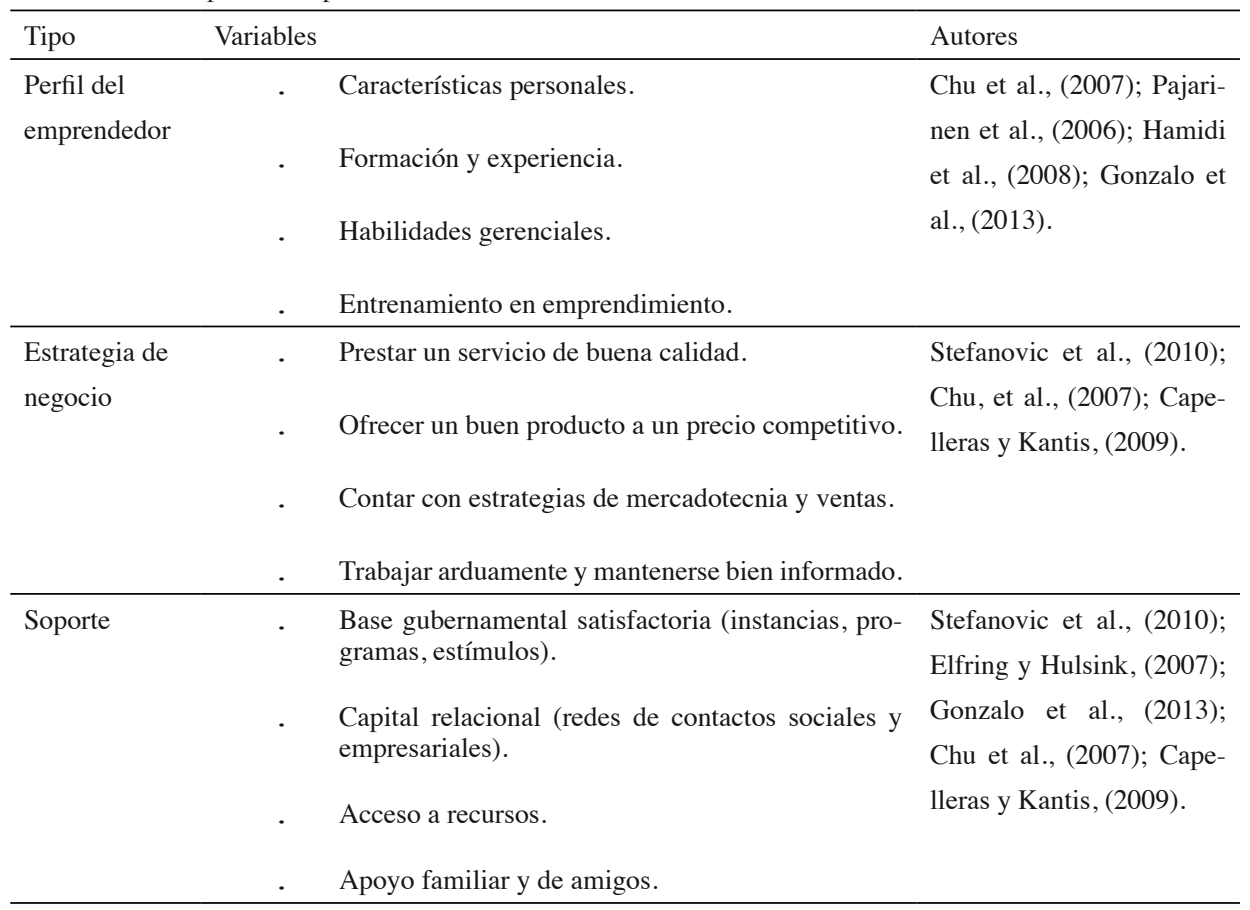

Fuente: Elaboración propia con base en la literatura revisada. 


\section{Desempeño empresarial}

El desempeño empresarial, es un referente del éxito compuesto por múltiples indicadores: financieros, operacionales y de mercado, que dan cuenta de los beneficios alcanzados mediante una actividad de negocio (Venkatraman y Ramanujam, 1986). Los factores que influyen en el mismo son difíciles de determinar. En la literatura sobre el emprendimiento, se refieren las motivaciones (Machmud y Sidharta, 2015; Charles et al., 2013); los factores de negocio (Lee et al., 2006; Coy et al., 2007); las competencias y el perfil del emprendedor (Barazandeh et al., 2015; Mitchelmore y Rowley, 2010; Benzing et al., 2009); así también los aspectos relacionados con la gestión y operación (Chandler y Hanks, 1994; Chaganti y Parasuraman, 1996), los cuáles han sido vinculados a la supervivencia, el crecimiento y la rentabilidad del negocio.

Con base en este soporte teórico, se conceptualiza el modelo que sustenta la presente investigación y se plantean las hipótesis respectivas. Ver Tabla 4.

Tabla 4

Hipótesis y soporte empírico

Hipótesis

H1: Las competencias para emprender (formación, experiencia, capacidades, habilidades) influyen positivamente sobre las motivaciones para emprender.

H2: Las competencias para emprender influyen positivamente sobre los factores de éxito para el emprendimiento (perfil del emprendedor, estrategias de negocio y soporte).

H3: Las competencias para emprender influyen positivamente sobre el desempeño empresarial (incremento en ventas, rentabilidad, crecimiento).

H4: Las motivaciones para emprender influyen positivamente sobre el desempeño empresarial.

H5: Los factores de éxito influyen positivamente sobre el desempeño empresarial.

H6: Las motivaciones para emprender influyen positivamente sobre los factores de éxito para el emprendimiento.

Benzing et al.,
Kapu, (2008). man, (1996).
Referencias complementarias

Fenton y Barry, (2014); Iglesias et al., (2016); Lee, Chang y Lim, (2005).

Chandler y Hanks, (1994); Chaganti y Parasura-

Mitchelmore y Rowley, (2010; 2013); Barazandeh et al, (2015); Lee et al., (2006).

Machmud y Sidharta, (2015); Charles et al., (2013); Eijdenberg, Masurel y Paas, (2015).

Lee et al., (2006); Mitchelmore y Rowley, (2010); Benzing et al., (2009); Coy et al., (2007).

Charles et al., (2013); Kuratko et al., (1997); Zimmerman y Chu, (2013); Stefanovic et al., (2010); al., (2009); Chu, et al., (2007); Yalcin y

Fuente: Elaboración propia. 


\section{Metodología}

La investigación es cuantitativa de tipo transversal causal; tiene como objetivo de estudio a los emprendedores de las micro, pequeñas y medianas empresas (MiPymes) del Estado de Querétaro, por considerar que el mayor surgimiento de emprendimientos se da en este tamaño de empresas. La selección de la muestra se hizo considerando el número de empleados, referente válido para clasificar a las organizaciones.

Bajo esta consideración y tomando en cuenta la información de la población objeto de estudio, de los sectores que conforman mayormente el aparato productivo del Estado de Querétaro, el tamaño de muestra estimado fue de 339 empresas con un nivel de confianza del $95 \%$ y $.05 \%$ de error. Esto equivale a 319 microempresas, 16 pequeñas y 4 medianas. El tamaño de muestra alcanzado fue de 447 empresas. De las encuestas levantadas, se eliminaron 18 por encontrarse información incompleta y presentar problemas de llenado, quedando entonces constituida la muestra por 429 empresas: 393 microempresas, 30 pequeñas y 6 medianas. Esto bajo un muestreo no probabilístico de conveniencia, con base en el interés de los emprendedores de participar en el estudio.

La recolección de datos se llevó a cabo en la zona metropolitana del Estado de Querétaro, la cual incluye a los municipios de Querétaro, Corregidora, El Marqués y Pedro Escobedo. En su conjunto integran el $68.7 \%$ de las unidades productivas. Para lo cual, se aplicó un cuestionario de 63 ítems, auto administrado a emprendedores en las instalaciones de su empresa, por encuestadores previamente capacitados; en el cual se integran cuestionamientos sobre aspectos generales y demográficos, al igual que otros sobre antecedentes familiares de emprendimiento y aquellos que se supone contribuyen al emprendimiento y éxito empresarial, éste último medido por el desempeño. El llenado de la información, se hizo en su mayoría por el dueño o propietario de la empresa (93\%) y el resto por el encargado de la misma, que principalmente resultó ser un familiar directo, esposa o hijos (5.3\%).

Para explorar los factores que contribuyen al emprendimiento y éxito empresarial, se revisaron investigaciones previas. Se definieron cómo constructos de estudio, las motivaciones, las competencias, los factores de éxito y el desempeño empresarial. Otros modelos, por ejemplo GEM (Global Entrepreneurship Monitor) incluyen en su análisis las intenciones o aspiraciones para emprender. No se introdujeron en el modelo propuesto, porque son un referente del potencial y la calidad del emprendimiento, lo cual favorece más la identificación de los tipos de emprendedores y está fuera del alcance de esta investigación. La selección de los ítems se hizo, tomando en cuenta las dimensiones y variables usadas para representar cada constructo, la confiabilidad y validez de los indicadores, y la calidad de los resultados obtenidos. A partir de allí, se diseñó la escala de medición y se llevó a cabo el proceso de validación aparente y de contenido con expertos en el área y una prueba piloto a 30 emprendedores. Se hicieron 
algunos ajustes de redacción y se procedió a su aplicación con la ayuda de los encuestadores.

Por lo que toca a los ítems, se utilizaron algunos de las escalas desarrolladas por Chu y Katsioloudes (2001); Chu et al.,(2007) y Stefanovic et al.,(2010) las cuales abordan aspectos relacionados con la motivación, el éxito y los grupos de influencia. Igual, otros de Venkatraman y Ramanujan (1986) para el desempeño empresarial que consideran: crecimiento, ventas y rentabilidad. Se emplearon también ítems de Olmos et al., (2014); De Paco, et al., (2011) y Amorós et al., (2011) relacionados con las competencias para emprender. Y se diseñaron algunos más exprofeso para la investigación. En todos los casos, se adaptaron al contexto local y se midieron mediante una escala Likert de 7 puntos de totalmente de acuerdo a totalmente en desacuerdo.

Con el fin de tener una primera aproximación a los factores y las variables que forman parte de este estudio, se llevó a cabo un análisis factorial exploratorio de componentes principales con rotación varimax. Se identificaron 4 factores, los cuales explican el $65.549 \%$ de la varianza total. Esto previa eliminación de las variables con cargas factoriales, menores a 6 y los ítems que cargaban en dos factores. El modelo de medida quedó integrado por 18 de las 49 variables exploradas. Las competencias bajo una estructura bidimensional y los otros constructos unidimensional, lo que llevó a plantear un modelo de medida de segundo orden.

Para evaluar si las variables observables miden correctamente los conceptos teóricos, se utilizó el análisis de fiabilidad y validez. Esta evaluación incluye la fiabilidad de los ítems, del constructo y la validez convergente y discriminante; para lo cual se utilizó el análisis factorial confirmatorio (AFC) con estimación de máxima verosimilitud mediante el software SPSS Amos. Los valores obtenidos del AFC muestran un buen ajuste de la estructura factorial propuesta y niveles de ajuste del modelo de medida apropiados. Las cargas estandarizadas son todas significativas y exhiben valores superiores a .6 (p<0.001) (Bagozzi y Yi, 1988); con lo que se asegura que la varianza compartida entre el constructo y los indicadores es mayor que la varianza de error (Fornell y Larcker, 1981; Hair et al., 2004). Los valores de ajuste del AFC, están también dentro de parámetros aceptables (Bentler y Bonett, 1980; Hu y Bentler, 1995). Ver Tabla 5.

En seguida, se procedió a estimar la fiabilidad del constructo a través de la evaluación de la consistencia interna, para lo cual se utilizaron el alfa de Cronbach y el índice de fiabilidad compuesta (IFC). Cómo regla general, los índices de fiabilidad no deben ser menores a 0.70. Los resultados de estos indicadores superan el umbral mínimo de 0.70 en cada una las dimensiones estudiadas (Nunnally y Bernstein, 1994; Hair, et al., 2004). En cuanto, a la validez convergente calculada con la varianza media extraída (AVE), los valores de AVE de las variables analizadas son mayores al mínimo sugerido de .5 (Fornell y Larcker, 1981). 
Tabla 5

Resultados de Fiabilidad y validez

\begin{tabular}{|c|c|c|c|c|c|c|}
\hline Factor & Variables & Indicador & Cargas & Alfa Cron. & IFC & AVE \\
\hline \multirow[t]{5}{*}{ Motivaciones } & Deseo de independencia & MOEMP1 & .712 & .821 & .8571 & .5460 \\
\hline & Alcanzar nuevos retos & MOEMP2 & .744 & & & \\
\hline & $\begin{array}{l}\text { Cumplir con un sueño o } \\
\text { meta personal }\end{array}$ & MOEMP3 & .757 & & & \\
\hline & $\begin{array}{l}\text { Oportunidad de realizar } \\
\text { ideas propias }\end{array}$ & MOEMP4 & .785 & & & \\
\hline & Mejor ingreso económico & MOEMP8 & .693 & & & \\
\hline \multirow[t]{2}{*}{ Competencias } & Capacidades y habilidades & HABCAP & .799 & .812 & .7116 & .5538 \\
\hline & Formación y experiencia & FOYEX & .685 & & & \\
\hline \multirow[t]{4}{*}{ Capacidades y } & Confianza en sí mismo & HACAP9 & .745 & & & \\
\hline & $\begin{array}{l}\text { Creatividad y perseve- } \\
\text { rancia }\end{array}$ & HACAP10 & .719 & & & \\
\hline & Persuasión y constancia & HACAP11 & .710 & & & \\
\hline & Compromiso por el logro & HACAP13 & .751 & & & \\
\hline \multirow{3}{*}{$\begin{array}{ll}\text { - } & \text { Formación y } \\
\text { experiencia en } \\
\text { emprendimiento }\end{array}$} & $\begin{array}{l}\text { Conocimiento del negocio } \\
\text { deseado }\end{array}$ & FOEX116 & .679 & & & \\
\hline & Experiencia laboral previa & FOEX217 & .729 & & & \\
\hline & $\begin{array}{l}\text { Formación adicional en } \\
\text { negocios }\end{array}$ & FOEX318 & .742 & & & \\
\hline \multirow[t]{3}{*}{ Factores de éxito } & Buen servicio al cliente & FAEXIT6 & .713 & .766 & .7658 & .5231 \\
\hline & $\begin{array}{l}\text { Establecimiento de es- } \\
\text { trategias de marketing y } \\
\text { ventas }\end{array}$ & FAEXIT7 & .795 & & & \\
\hline & $\begin{array}{l}\text { Redes personales, sociales } \\
\text { y de trabajo vinculadas al } \\
\text { ámbito empresarial }\end{array}$ & FAEXIT9 & .655 & & & \\
\hline \multirow[t]{3}{*}{$\begin{array}{l}\text { Desempeño } \\
\text { empresarial }\end{array}$} & $\begin{array}{l}\text { Satisfacción con nivel de } \\
\text { crecimiento }\end{array}$ & DECRE1 & .644 & .769 & .7719 & .5319 \\
\hline & $\begin{array}{l}\text { Satisfacción con incre- } \\
\text { mento en ventas }\end{array}$ & DEREN2 & .742 & & & \\
\hline & $\begin{array}{l}\text { Satisfacción con nivel de } \\
\text { rentabilidad }\end{array}$ & DEVEN3 & .794 & & & \\
\hline
\end{tabular}

Nota: AFC: $(\mathrm{CMIN}=239.816 ; \mathrm{DF}=127 ; \mathrm{CMIN} / \mathrm{DF}=1.888 ; \mathrm{NFI}=.919 ; \mathrm{TLI}=.952 ; \mathrm{CFI}=.960 ; \mathrm{RMSEA}=.046$, $* * *=\mathrm{p}<0.001)$.

Fuente: Elaboración propia. 
Respecto de la validez discriminante, la cual es el referente de la diferencia teórica existente entre los distintos constructos, se estimó la raíz cuadrada del AVE de cada constructo, criterio sugerido por Fornell y Larcker (1981). Se verificó que los valores del AVE fueran superiores a las correlaciones con cualquier otro constructo. La Tabla 6, exhibe los estadísticos encontrados. Según estos resultados, la condición de discriminación se cumple para todos los constructos.

Tabla 6

Indicadores de validez discriminante

\begin{tabular}{lllll}
\hline Factores & Motivaciones & Competencias & Factores de éxito & Desempeño empresarial \\
Motivaciones & .7389 & & \\
Competencias & .659 & .7441 & \\
Factores de éxito & .104 & .272 & .7232 \\
Desempeño empresarial & .053 & .463 & .370 & .7293 \\
\hline
\end{tabular}

Nota: La diagonal representa la varianza media extraída (AVE) mientras que por debajo de la diagonal se muestran las estimaciones de las correlaciones de los factores.

Fuente: Elaboración propia.

\section{Resultados}

La Tabla 7, resume la información relacionada con la muestra. En ella se observan, porcentajes más grandes de hombres participando en emprendimientos; igualmente que son las personas mayores de 35 años las que optan por su independencia y crean su propio negocio. El porcentaje más significativo de emprendedores sólo tiene estudios de bachillerato, carrera técnica o educación básica (primaria y secundaria). Únicamente, el 39.5\% cuenta con estudios universitarios y de posgrado. El emprendimiento se presenta mayormente en personas casadas, sin antecedentes familiares en el desarrollo de actividades de este tipo (69.4\%). Y el mayor estímulo para emprender proviene de sus familiares $(42.1 \%)$. En cuanto a la unidad muestral, está integrada por micronegocios $(91.5 \%)$, pequeñas $(7 \%)$ y medianas empresas $(1.5 \%)$ de los sectores de servicios, comercio y manufactura. Siendo los hombres lo que emprenden mayormente en el sector de manufactura, mientras las mujeres lo hacen en el de servicios y comercio. La mayoría de las empresas de los emprendedores han estado funcionando en el mercado por más de 5 años. 
Tabla 7

Características de la muestra y la unidad muestral

\begin{tabular}{|c|c|c|c|c|c|c|}
\hline \multirow[t]{2}{*}{ Género } & \multicolumn{4}{|l|}{ Hombres } & \multicolumn{2}{|l|}{ Mujeres } \\
\hline & \multicolumn{4}{|l|}{$74.7 \%$} & \multicolumn{2}{|l|}{$25.3 \%$} \\
\hline \multirow[t]{2}{*}{ Edad } & \multicolumn{2}{|l|}{$>35$ años } & \multicolumn{2}{|l|}{25 a 35 años } & \multicolumn{2}{|l|}{$<25$ años } \\
\hline & $59.7 \%$ & & \multicolumn{2}{|l|}{$30.5 \%$} & \multicolumn{2}{|l|}{$9.8 \%$} \\
\hline \multirow[t]{2}{*}{ Nivel de estudios } & Posgrado & Licen & ingeniería & $\begin{array}{l}\text { Bachillerato o } \\
\text { carrera técnica }\end{array}$ & $\begin{array}{l}\text { Educación } \\
\text { básica }\end{array}$ & $\begin{array}{l}\text { Sin estu- } \\
\text { dios }\end{array}$ \\
\hline & $19.4 \%$ & 20.19 & & $30.9 \%$ & $28.4 \%$ & $1.2 \%$ \\
\hline Estado civil al & \multicolumn{2}{|l|}{ Casados } & \multicolumn{2}{|l|}{ Solteros } & \multicolumn{2}{|c|}{ Divorciados/Viudos } \\
\hline emprender & \multicolumn{2}{|l|}{$56.7 \%$} & \multicolumn{2}{|l|}{$37.5 \%$} & \multicolumn{2}{|l|}{$5.8 \%$} \\
\hline Antecedentes & \multicolumn{4}{|l|}{$\mathrm{Si}$} & \multicolumn{2}{|l|}{ No } \\
\hline $\begin{array}{l}\text { familiares de } \\
\text { emprendimiento }\end{array}$ & \multicolumn{4}{|l|}{$30.6 \%$} & \multicolumn{2}{|l|}{$69.4 \%$} \\
\hline Soporte para & \multicolumn{2}{|l|}{ Familiares } & \multicolumn{2}{|l|}{ Amigos } & \multicolumn{2}{|c|}{ Colegas de trabajo } \\
\hline emprender & \multicolumn{2}{|l|}{$42.1 \%$} & \multicolumn{2}{|l|}{$30.6 \%$} & \multicolumn{2}{|l|}{$27.3 \%$} \\
\hline \multirow[t]{2}{*}{ Tipo de empresa } & \multicolumn{2}{|l|}{ Servicios } & \multicolumn{2}{|l|}{ Manufactura } & \multicolumn{2}{|l|}{ Comercio } \\
\hline & \multicolumn{2}{|l|}{$39.0 \%$} & \multicolumn{2}{|l|}{$37.7 \%$} & \multicolumn{2}{|l|}{$23.3 \%$} \\
\hline \multirow[t]{2}{*}{ Edad } & Jóvenes (< & años) & & & Maduras (>5 & iños) \\
\hline & $35.8 \%$ & & & & $64.2 \%$ & \\
\hline Tamaño & Micro & & Pequeña & & Mediana & \\
\hline & 1 a $10 \mathrm{emp}$ & $\operatorname{ados}$ & 11 a $50 \mathrm{emp}$ & $\operatorname{ados}$ & $>50 \mathrm{empl}$ & idos \\
\hline & $91.5 \%$ & & $7 \%$ & & $1.5 \%$ & \\
\hline
\end{tabular}

Fuente: Elaboración propia.

En lo que concierne a los resultados del modelo conceptual propuesto, con el cual se busca probar los supuestos planteados, la valoración del ajuste del modelo de acuerdo a los resultados obtenidos y a los parámetros de referencia establecidos reflejan que es satisfactorio, es decir las estructuras teóricas y los datos empíricos sugieren un buen nivel de ajuste $(\mathrm{CMIN}=239.816 ; \mathrm{DF}=127 ; \mathrm{CMIN} / \mathrm{DF}=1.888 ; \mathrm{NFI}=.919 ; \mathrm{TLI}=952 ; \mathrm{CFI}=.960 ; \mathrm{RMSEA}=$ $.046, * * *=\mathrm{p}<0.001)$. Los valores de NFI y CFI son mayores de 9 (Bentler y Bonett, 1980). La razón de chi cuadrado sobre los grados de libertad presenta valores inferiores a 2 . El índice RMSEA es también menor a 0.06 (Hu y Bentler, 1995).

Con relación al contraste de hipótesis, los resultados obtenidos de $\mathrm{H} 1(\beta=0.659, \mathrm{p}<0.001)$ muestran que las competencias para emprender tienen efectos estadísticamente significativos y positivos sobre las motivaciones para emprender. Esto prueba la relación e influencia de las competencias sobre las motivaciones para autoemplearse e iniciar una empresa. En cuanto a 
la hipótesis $\mathrm{H} 2$ ( $\beta=0.419, \mathrm{p}<0.001$ ), los hallazgos demuestran que las competencias para emprender influyen positiva y significativamente sobre los factores de éxito que contribuyen al emprendimiento. En este sentido, se confirma el papel preponderante que asumen las competencias en el ejercicio de las tareas críticas para llevar un emprendimiento a buen término.

Respecto de la hipótesis H3, se valida el impacto que tienen las competencias para emprender sobre el desempeño empresarial $(\beta=.605, \mathrm{p}<0.001)$. Las implicaciones de estos hallazgos sugieren que el desempeño empresarial, medido por la rentabilidad, crecimiento y ventas, depende en gran medida de las habilidades y capacidades del emprendedor. Por lo que corresponde a la hipótesis $\mathrm{H} 4$, los resultados alcanzados $(\beta=-.306, \mathrm{p}=0.006)$ revelan que las motivaciones tienen un efecto significativo pero negativo sobre el desempeño empresarial. Esto significa que las personas que están altamente motivadas por aspectos de logro personal o económico tendrán menores niveles de desempeño empresarial. En lo que hace a la hipótesis $\mathrm{H} 5$, los estimados obtenidos $(\beta=.222, \mathrm{p}=0.002)$ reflejan que el peso de los factores de éxito en el desempeño empresarial es igualmente significativo. Esto confirma que determinadas cuestiones de negocio asumen una función medular en el logro de los resultados organizacionales. Finalmente, los resultados de la hipótesis H6 ( $\beta=-.224$, p= $0.029)$ muestran que las motivaciones influyen de manera significativa, pero negativa sobre los factores de éxito que contribuyen al emprendimiento, lo cual sugiere que un nivel alto de este tipo de motivaciones no necesariamente estará asociado a mejores factores de éxito. La Tabla 8 exhibe los resultados del modelo estructural y las pruebas de hipótesis respectivas.

Tabla 8

Resultados del modelo estructural

\begin{tabular}{lccc}
\hline Hipótesis & Valores $\beta$ & Sig. & Prueba de hipótesis \\
\hline H1 Competencias --- > Motivaciones & $.659 * * *$ & .000 & Aceptada \\
H2 Competencias --- > Factores de éxito & $.419 * * *$ & .000 & Aceptada \\
H3 Competencias --- > Desempeño empresa- & $.605 * * *$ & .000 & Aceptada \\
rial & & & \\
H4 Motivaciones --- > Desempeño empresarial & $-.306 * *$ & .006 & Rechazada \\
H5 Factores de éxito --- > Desempeño empre- & $.222 * *$ & .002 & Aceptada \\
sarial & & & \\
H6 Motivaciones --- > Factores de éxito & $-.224 *$ & .029 & Rechazada \\
\hline
\end{tabular}

Nota: *: p < 0.05,**: $\mathrm{p}<0.01, * * *: \mathrm{p}<0.001$

Fuente: Elaboración propia. 


\section{Discusión}

Los hallazgos alcanzados dan evidencia de una relación significativa entre las motivaciones, las competencias; así también los factores de éxito para el emprendimiento con el desempeño empresarial. Se prueba que las competencias para emprender influyen en las motivaciones para iniciar una empresa, H1. En este sentido, los resultados son coincidentes a los de Fenton y Barry, (2014); Iglesias et al., (2016); Lee et al., (2005) en lo que se refiere al reconocimiento de la importancia de las competencias en la predisposición a emprender y la promoción de su desarrollo. Esto hace suponer, que a mayor formación y exposición a entornos de emprendimiento, más fortalezas tendrá la persona que emprende y mayor probabilidad habrá de qué considere el independizarse y emprenda exitosamente. De ahí, que la formación para el emprendimiento, debiera configurarse para empoderar al potencial emprendedor, reforzar la percepción sobre de sus propias capacidades; enfatizar los aspectos que facilitan la actividad emprendedora y sortear aquellos que la obstaculizan.

De igual forma, se halla que las competencias para emprender influyen en los factores de éxito que contribuyen al emprendimiento, H2. Las implicaciones de estos resultados evidencian que este cúmulo de saberes, habilidades y capacidades favorecen el que una persona pueda aprovechar las oportunidades, asumir riesgos y gestionar un negocio efectivamente; y de esta forma implementar estrategias; por ejemplo, de mercadotecnia y ventas que le ayuden a identificar su nicho de mercado y posicionar su producto. Asimismo, a estar orientado al cliente y a construir el capital relacional que le permita acceder a oportunidades de negocio; con lo cual reducirá su vulnerabilidad sobre todo en la etapa de iniciación y puesta en marcha. Otros investigadores han alcanzado resultados similares en cuanto a las competencias tanto emprendedoras como gerenciales e igual han identificado los mismos factores de éxito (Chandler y Hanks, 1994; Chaganti y Parasuraman, 1996; Stefanovic et al., 2010; Chu et al., 2007).

En lo que hace a la hipótesis H3, es visible que las competencias para emprender representan el factor de mayor peso en el éxito empresarial. Otros investigadores han tenido hallazgos similares con los mismos indicadores, especialmente en lo que hace a la educación y habilidades para emprender (Mitchelmore y Rowley, 2010; 2013; Lee et al., 2006; Barazandeh et al, 2015; Lee et al., 2005). De modo que, puede afirmarse que las competencias para emprender constituyen un pilar sólido sobre el que debieran apoyarse todos aquellos que deseen iniciarse en la aventura empresarial. Y queda claro, que entre mayores competencias tenga el emprendedor, menor será la probabilidad de fracaso de nuevas empresas. Lo anterior podría sugerir, que no es aconsejable emprender, si no se está preparado para reconocer las oportunidades, generar proyectos innovadores, definir un modelo de negocio, evaluar riesgos e integrarse a redes de proveeduría, servicios, emprendedores, entre otros.

Con respecto a la $\mathrm{H} 4$, que explora la influencia de las motivaciones para emprender en 
el desempeño empresarial, los resultados fueron significativos pero negativos y difieren de aquellos alcanzados en estudios empíricos previos, que han probado la existencia de un vínculo positivo y significativo (Machmud y Sidharta, 2015; Charles et al., 2013; Eijdenberg et al., 2015; Muralanda y Morales, 2016). En este caso, las motivaciones que resultaron más robustas para su incorporación al modelo estructural, fueron de tipo intrínseco de logro como el alcanzar nuevos retos, llevar a cabo ideas propias, cumplir con un sueño, deseo de independencia y obtener un mejor ingreso. Dados estos resultados, otros factores motivacionales, por ejemplo aquellos que afectan la situación económica y laboral del emprendedor, de regulación para abrir empresas, acceso a capital y/o apoyo gubernamental al emprendimiento, etc., pudiesen favorecer éste. Las motivaciones per sé parecen no ser el mejor predictor del desempeño empresarial.

En relación a la hipótesis $\mathrm{H} 5$, el efecto de los factores de éxito para el emprendimiento sobre el desempeño empresarial fue probado al resultar estadísticamente significativo, lo cual coincide con los hallazgos obtenidos por Lee et al., (2006); Mitchelmore y Rowley, (2010); Benzing et al., (2009); Coy et al., (2007); Stefanovic et al., (2010). Los alcances subyacentes, ponen de relieve la trascendencia de contar un modelo y estrategia de negocio, que guíe el actuar de la empresa y la creación de valor al cliente para anticipar el éxito y un buen desempeño, sobretodo en la etapa de arranque y estabilización de la empresa; y reducir así los altos índices de mortalidad empresarial. Desde esta perspectiva, es claro que bajo ciertos entornos y circunstancias, las habilidades del emprendedor serán decisivas en la definición del rumbo y futuro de la empresa.

Por otro lado la hipótesis H6, constata el efecto de las motivaciones sobre los factores de éxito para el emprendimiento, aunque su influencia es negativa. A la vista de estos resultados, las variables explicativas de los emprendimientos exitosos parecieran tener que ver poco con las motivaciones intrínsecas y más bien con otros aspectos motivacionales exógenos que pudiesen favorecen las oportunidades de negocio como el mercado, la incursión en determinada actividad empresarial y la capacidad para aprovecharlas. En este sentido, los resultados difieren a los encontrados en investigaciones previas en las cuales se ha probado el efecto positivo de la motivación en el éxito emprendedor (Charles et al., 2013; Kuratko et al., 1997; Zimmerman y Chu, 2013; Stefanovic et al., 2010; Benzing et al., 2009; Chu, et al., 2007; Yalcin y Kapu, 2008).

A la luz de otros estudios en México, estos hallazgos muestran similitudes respecto del vínculo positivo y significativo entre las competencias del emprendedor y los logros organizacionales (De la Garza, Zavala y López, 2017); la identificación de las razones por las que se emprende (Bueckmann et al., 2018; Partida, Cabrera y Villarreal, 2012); la educación y experiencia como motivadores para emprender (Bueckmann et al., 2018; Moska, Naranjo y Aguirre, 2015; Rehak et al., 2016) y su influencia en el crecimiento de la empresa (Partida et 
al., 2012). Igual, hay coincidencias en cuanto a los factores de éxito: el servicio al cliente, un buen producto a precio competitivo, habilidades gerenciales, estrategias de comercialización (Partida et al, 2012) y relaciones de negocio (Moska et al., 2015).

\section{Conclusiones}

De los resultados de la investigación se desprenden una serie de conclusiones. La primera de ellas, es la evidencia empírica sobre la importancia de las competencias para emprender, ya que para el caso de los emprendedores de Querétaro fueron el factor determinante en la decisión de iniciar una empresa, lo que los motivó a emprender y a manejar un negocio exitosamente. Investigaciones previas, han alcanzado resultados similares en otros contextos (Fenton y Barry, 2014; Iglesias et al., 2016; Lee et al, 2005; Chandler y Hanks, 1994; Chaganti y Parasuraman, 1996; Stefanovic et al., 2010; Chu et al., 2007).

De ahí que, para elevar el potencial emprendedor y la tasa de éxito en el emprendimiento del Estado, sea prioritario fortalecer la formación de talento emprendedor y estimular un entorno para el emprendimiento ad hoc a las condiciones que predominan en el Estado; que brinde al emprendedor el soporte tecnológico, de negocios y financiero que el mismo requiera. En estos las instituciones educativas y las instancias gubernamentales son los partícipes principales.

A partir de estos resultados, es posible también sugerir que los aspectos de formación y experiencia en emprendimiento, debieran ser un factor a considerar para el otorgamiento de créditos y subsidios al emprendedor, sobre todo aquellos destinados a apoyar la creación y operación de nuevas empresas, ya que pueden significar la diferencia entre el éxito o fracaso de una actividad de este tipo o representar un elemento costoso, que aunque conlleva un profundo aprendizaje puede influir en las decisiones futuras de incursionar o financiar una idea de negocio.

La segunda conclusión, es que las motivaciones de tipo personal fueron el principal impulso para emprender, lo que refleja que el emprendimiento tiene que ver más con un proyecto de vida y de desarrollo personal. Estudios preliminares muestran coincidencias con estos hallazgos (Chu et al., 2007; Stefanovic et al., 2010; Coduras et al., 2016). En consecuencia, identificar a personas con estas motivaciones desde etapas tempranas e incorporarlos a programas de formación y entornos de emprendimiento, pudiese ser una alternativa para potencializar las iniciativas empresariales e incrementar la tasa de efectividad. También, asumir que aunque se ha probado que las motivaciones son un precedente en la decisión de emprender su influencia en el desempeño no es aún concluyente.

La tercera y última, es que el éxito recae fuertemente en las cuestiones vinculadas al negocio. Otros trabajos de investigación igual han llegado a estas mismas conclusiones 
(Partida et al., 2012; Moska et al., 2015). Por lo que, para asegurar el mismo sea necesario canalizar todos los esfuerzos y recursos, a generar competencias que coadyuven a profundizar el conocimiento del negocio y la gestión. Así también, a establecer modelos y estrategias que favorezcan los aspectos de costos, diferenciación y mercado que apuntalen el crecimiento, rentabilidad y la consolidación de su empresa.

Dentro de las limitaciones de este estudio, están la no estratificación de la muestra por sector, el tipo de muestreo que fue no probabilístico y la selección de la muestra acotada al área metropolitana del Estado. Como áreas futuras de investigación, podrían sugerirse el explorar otros factores que pudiesen contribuir al emprendimiento, como el tipo de emprendedores, iniciativas de negocio, ecosistemas para el emprendimiento y programas de fomento al emprendedor, por mencionar algunos.

\section{Referencias}

Acs, Z. y Szerb, L. (2011). Global Entrepreneurship and Development Index 2011. Cheltenham, UK: Edward Elgar Publishing.

Amit, S. y Muller, E. (1995). Push and Pull entrepreneurship. Journal of Small Business \& Entrepreneurship, 12(4), 64-80, http://dx.doi.org/10.1080/08276331.1995.10600505.

Amorós, J., Fernández, C. y Tapia, J. (2011). Quantifying the relationship between entrepreneurship and competitiveness development stages in Latin America. International Entrepreneurship and Management Journal, 8(3), 249-270. http://dx.doi.org/10.1007/ s11365-010-0165-9.

Bagozzi, R. y Yi, Y. (1988). On the evaluation of structural equation model. Journal of the Academy of Marketing Science, Spring (16), 74-94. http://dx.doi.org/10.1007/ BF02723327.

Barazandeh, M., Parvizian, K., Alizadeh, M, y Khosrav, S. (2015). Investigating the effect of entrepreneurial competencies on business performance among early stage entrepreneurs. Global Entrepreneurship Monitor. Journal of Global Entrepreneurship Research, 5(1). http://dx.doi.org/10.1186/s40497-015-0037-4.

Bentler, P. y Bonett, D. (1980). Significance tests and goodness of fit in the analysis of covariance structures. Psychological Bulletin, 88(3), 588-606. http://dx.doi. org/10.1037/00332909.88.3. 588.

Benzing, C., Chu, H. y Callanan, G. (2005). A regional comparison of the motivation and problems of Vietnamese entrepreneurs. Journal of Developmental Entrepreneurship 10(01), 3-27. http://dx.doi.org/10.1142/s1084946705000033.

Benzing, C., Chu, H. y Kara, O. (2009). Entrepreneurs in Turkey: a factor analysis of motivations success factors and problems. Journal of Small Business Management, 47(1), 58-91. http://dx.doi.org/10.1111/j.1540-627X.2008.00262.x. 
Bueckmann, D., Barrera, P., Alonso, P., Rehak, J. y Castro, D. (2018). El Emprendimiento en el Estado de Querétaro. Análisis de los datos del Global Entrepreneurship Monitor 20172018. ITESM, 1-66 [consultado 15 Ago 2018]. Disponible en: https://www.researchgate. net/publication /330293180_Queretaro_State_Global_Entrepreneurship_Monitor_Report_2017_2018\#fullTextFileContent.

Busenitz, L., West, G., Shepherd, D., Nelson, T., Chandler, G. y Zacharakis, A. (2003). Entrepreneurship research in emergence: Past trends and future directions. Journal of Management, 29(3), 285-308. http://dx .doi.org/10.1016/S0149-2063(03)00013-8.

Cachon, J., Codina, J., Wellmann, C., Mc Graw, E. y Myers, D. (2013). Entrepreneurial Motives and Performance. Evidence from North America. Journal of Management Policy and Practice, 14 (3), 50-77 [consultado 13 Jul 2018]. Disponible en: https://www. researchgate.net/publication $/ 260407210$.

Canales, R., Román, Y. y Ovaldo, W. (2017). Emprendimiento de la población joven en México. Una perspectiva crítica. Entreciencias: Diálogos en la Sociedad del Conocimiento, 5(12), 1-17. http://dx.doi.org//10.21933/J.EDSC.2017.12.211.

Capelleras, J. y Kantis, D. (2009). Nuevas empresas en América Latina: factores que favorecen su rápido crecimiento. (1er Ed.). Barcelona: Universidad Autónoma de Barcelona y Universidad Nacional de General Sarmiento.

Carsrud, A. y Brännback, M. (2011). Entrepreneurial Motivations: What Do We Still Need to Know? Journal of Small Business Management, 49(1), 9-26. http://dx.doi.org/10.1111/ j.1540-627X. 2010.00312.x.

Castillo, C. y Orlando, J. (2008). Círculo virtuoso de la educación empresarial: Una propuesta de modelo para el desarrollo de un tejido empresarial de calidad. Revista Escuela de Administración de Negocios, mayo-agosto (63), 23-39. http://dx.doi. org/10.21158/01208160.n63.2008.439.

Chaganti, R. y Parasuraman, S. (1996). A study of the impacts of gender on business performance and management patterns in small businesses. Entrepreneurship Theory and Practice, 21(2), 73-75. http://dx.doi.org/10.1177/104225879602100205.

Chandler, G. y Hanks, S. (1994). Market attractiveness, resource-based capabilities, venture strategy and venture performance. Journal of Business Venturing, 9(4), 331-349. http://dx.doi.org/10.10 16/ 0883-9026(94)90011-6.

Charles, J., Chacon, J., Barajan, J. Wellman, C. y Myers, D. (2013). Entrepreneurial Motives and Performance: Evidence from North America. Journal of Management Policy and Practice, 14(3), 50-77 [consultado 2 Jul 2018]. Disponible en: https://www.researchgate.net/ publication/ 260407210_Entrepreneurial_Motives_and_Performance_ Evidence_from_North_America.

Chu, H., Benzing, C. y McGee, C. (2007). Ghanaian and Kenyan entrepreneurs: a comparative analysis of their motivations, success characteristics and problems. Journal of Development Entrepreneurship, 12(3), 295-322. http://dx.doi.org/10.1142/ S1084946707000691. 
Chu, H. y Katsioloudes, M. (2001). Cultural context in the Vietnamese American entrepreneurial experience. Journal of Transnational Management Development, 7(2), 3746. http://dx.doi.org/10.1300/J130v07n02_04.

Coduras, A., Sainz, J. y Ruiz, J. (2016). Measuring readiness for entrepreneurship: An information tool proposal. Journal of Innovation \& Knowledge, 1(2), 99-108. http://dx .doi. org/10.1016/j.jik.2016.02.003.

Colombo, M. y Grilli, L. (2005). Founder's human capital and the growth of new technology-based firms: A competence-based view. Research Policy, 34(6), 795-816. http:// dx .doi.org/10.1016/j.respol.2005.03.010.

Consortium for Entrepreneurship Education (2008). Entrepreneurship everywhere: A Guide to Resources and Models for Entrepreneurship Education. 1-116 [consultado 20 Jun 2018]. Disponible en: http://www.arc.gov/programs/reginit/entevery/entrepix.htm.

Coy, S., Shipley, M., Omer, K. y Khan, R. (2007). Factors Contributory to Success: A Study of Pakistan's Small Business Owners. Journal of Developmental Entrepreneurship, 12(2), 181-198. http://dx.doi.org/ 0.1142/S1084946707000617.

De la Garza, M., Zavala, M. y López, J. (2017). Competencias del emprendedor y su impacto en el desempeño organizacional. Universidad \& Empresa, 19(33), 53-74. http:// dx.doi.org/10.12804/revistas.urosario.edu.co/empresa/a.4811.

De Paco, A., Ferreira, J., Raposo, M., Gouveia, R. y Dinis, A. (2011). Behaviours and entrepreneurial intention: Empirical findings about secondary students. Journal of International Entrepreneurship, 9(1), 20-38. http://dx.doi.org/10.1007/s10843-010-0071-9.

Díaz, J., Hernández, R. y Roldán, J. (2011). A structural model of the antecedents to entrepreneurial capacity. International Small Business Journal, 30(8), 850-872. http://dx.doi. org/10.1177/0266242610385263.

Dubini, P. (1988). The influence of motivations and environment on business start-ups: Some hints for public policies. Journal of Business Venturing, 4(1), 11-26. http://dx.doi. org/10.1016/0883-90268990031-1.

Edelman, L., Manolova, T., Shirokova, G. y Tsukanova, T. (2016). The impact of family support on young entrepreneurs' start-up activities. Journal of Business Venturing, 31(4), 428-448. http://dx.doi.org/10.1016/j.jbusvent.2016.04.003.

Eijdenberg, E., Masurel, E. y Paas, L. (2015). Entrepreneurial motivation and small business growth in Rwanda. Journal of Entrepreneurship in Emerging Economies, 7(3) 212-240. http://dx.doi.org/10.1108/JEEE-01-2015-0004.

Elfring, T. y Hulsink, W. (2007). Networking by entrepreneurs: patterns of information in emerging organizations. Organization studies, 28(12), 1849-1872. http://dx.doi.org/ $10.1177 / 0170840607078719$.

Failure Institute, (2016). El fracaso en Startups tecnológicas en México, 1-88 [consultado 26 Jul 2018]. Disponible en: https://thefailureinstitute.com/wp-content/uploads/2016/09/ Tech-Startup-Fail ure-Research-final.pdf.

Fenton, M. y Barry, A. (2014). Breathing space-graduate entrepreneurs perspectives of en- 
trepreneurship education in higher education. Education + Training, 56(8/9), 733-744. http://dx .doi.org/10.1108/ET-05-2014-0051.

Fornell, C. y Larcker, D. (1981). Evaluating structural equations models with unobservable variables and measurement error. Journal of Marketing Research, 18(1), 39-50. http:// dx.doi.org/ 10.2307/3151312.

Fuentelsaz, L., González, C., Maícas, J. y Montero, J. (2015). How different formal institutions affect opportunity and necessity entrepreneurship. BRQ Business Research Quartely, 18(4), 246-258. http://dx.doi.org/10.1016/j.brq.2015.02.001.

Gómez, M. y Zatizábal, K. (2011). Educación en emprendimiento. Fortalecimiento de competencias emprendedoras en la Pontificia Universidad Javeriana Cali. Economía, Gestión y Desarrollo, Junio (111), 121-155 [consultado 19 Jun 2018]. Disponible en: http://revistaeconomia. puj.edu.co/html/articulos/Numero_11/SATIZABAL.pdf.

Gonzalo, M., Federico, J. y Kantis, H. (2013). Crecimiento y adaptación en un contexto de crisis internacional: los casos de tres empresas jóvenes dinámicas argentinas. Techint, Enero-Abril (342), 117-134 [consultado 7 Ago 2018]. Disponible en: https://www.Researchgate.net/ publication/303207225.

Guerra, O., Hernández, D. y Triviño, G. (2015). Incubadora de empresas: Vía para el emprendimiento en las universidades. Revista Universidad y Sociedad, 7(1), 110-114 [consultado 24 Ago 2018]. Disponible en: http://scielo.sld.cu/scielo.php?script=sci_arttext\&pid=S2218-3620201500010 0016.

Hair, J., Anderson, R., Tatham, R. y Black, W. (2004). Análisis Multivariante. (1er Ed.). Madrid: Pearson Prentice Hall Internacional.

Hamidi, D., Wennberg, K. y Berglund, H. (2008). Creativity in entrepreneurship education. Journal of Small Business and Enterprise Development, 15(2), 304-320. http://dx.doi. org/10.1108/14626000810871691.

Hamilton, B. (2000). Does entrepreneurship pay? An empirical analysis of the returns to self-employment. Journal of Political Economy 108(3), 604-631. http://dx.doi.org/ $10.1086 / 262131$.

Hisrich, R., Peters, M. y Shepherd, D. (2013). Entrepreneurship. (7th Ed.). New York: Mc Graw Hill.

Hmieleski, K., Corbett, A. y Baron, R. (2013). Entrepreneur's improvisational behavior and firm performance: A study of dispositional and environmental moderators. Strategic Entrepreneurship Journal, 7(2), 138-150. http://dx.doi.org/10.1002/sej.1143.

Hu, L. y Bentler, P. (1995). Evaluating model fit. In R. H. Hoyle (Ed.), Structural equation modeling. Concepts, issues, and applications, 76-99. London: Sage.

Iglesias, P., Jambrino, J., Peñafiel, A. y Kokash, H. (2016). Impact of entrepreneurship programmes on university students. Education + Training, 58(2), 209-228. http://dx.doi. org/10.1108/et-01-2015-0004.

Instituto Nacional de Estadística y Geografía (2014). Censos Económicos. INEGI [consultado 18 Jun 2018]. Disponible en: https://www.inegi.org.mx. 
Instituto Nacional de Estadística y Geografía (2019). Directorio Estadístico Nacional de Unidades Económicas, DENUE Interactivo [consultado 18 Jun 2018]. Disponible en: https://www.inegi.org.mx/app/mapa/denue/default.aspx.

Johansen, V. (2010). Entrepreneurship education and entrepreneurial activity. International Journal of Entrepreneurship and Small Business, 9(1), 74-85. http://dx .doi.org/10.1504/ IJESB.2010.029507.

Kantis, H., Angelelli, P. y Koenig, V. (2004). Desarrollo Emprendedor. América Latina y la experiencia internacional. Banco Interamericano de Desarrollo, 1-287 [consultado 7 Ago 2018]. Disponible en: https://publications.iadb.org/handle/11319/442?locale-attribute $=$ es $\&$.

Kuratko, D., Hornsby, J. y Naffziger, D. (1997). An examination of owner's goals in sustaining entrepreneurship. Journal of Small Business Management, 35(1), 24-33 [consultado 15 Sep 2018]. Disponible en: https:/www.questia.com/library/journal/1G1-19360491/ an-examination-of-owner-s-goals-in-sustaining-entrepreneurship.

Lee, S., Chang, D. y Lim, S. (2005). Impact of entrepreneurship education: a comparative study of the US and Korea. International Entrepreneurship and Management Journal, 1(1), 27-43. http://dx.doi.org/10.1007/s11365-005-6674-2.

Lee, S., Lim S., Pathank, R., Chang, D. y Li, W. (2006). Influences on students attitudes toward entrepreneurship: a multi-country study. International Entrepreneurship Management Journal, 2(3), 351-366. http://dx.doi.org/10.1007/s11365-006-0003-2.

Léger, C. y Kaloussis, G. (2006). Entrepreneurship and Engineers: how to develop the spirit? Estudios de economía aplicada, 24(2), 525-544 [consultado 12 Sep 2018]. Disponible en: http://www.revista-eea.net/volumen.php?Id=60\&vol=24\&ref=2.

Liñán, F. y Chen, Y. (2009). Development and cross-cultural application of a specific instrument to measure entrepreneurial intentions. Entrepreneurship Theory and Practice, 33(3) 593-617. http://dx.doi.org/10.1111/j.1540-6520.2009.00318.x.

Machmud, S., y Sidharta, I. (2015). Motivation and Business Performance of SMEs in the SUCI Clothing Center, Bandung Indonesia, Business \& Economics Review, 25(2), 6378 [consultado 18 Sep 2018]. Disponible en: https://www.dlsu.edu.ph/wp-content/uploads/2019/03/5machmud-0206 16.pdf.

Martínez, F. y Carmona, G. (2009). Aproximación al concepto de competencias emprendedoras: valor social e implicaciones educativas. Revista Iberoamericana sobre Calidad, Eficacia y Cambio en Educación, 7(3), 82-98 [consultado 17 Sep 2018]. Disponible en: http://hdl.handle.Net /10486/661237.

Marulanda, F., Montoya, I. y Vélez, J. (2014b). Teorías motivacionales en el estudio del emprendimiento. Pensamiento \& Gestión, Enero-Junio (36), 206-238 [consultado 19 Sep 2018]. Disponible en: http://dx.doi.org/10.14482/pege.36.5571.

Marulanda, F. y Morales, S. (2016). Entorno y motivaciones para emprender. Rev. Esc. Adm. Neg., Julio-Diciembre (81), 12-28 [consultado 12 Sep 2018]. Disponible en: http://dx. doi.org/10.21158/ 01208160.n81.2016.1556. 
Mitchelmore, S. y Rowley, J. (2010). Entrepreneurial competencies: a literature review and development agenda. International Journal of Entrepreneurial Behaviour \& Research, 16(2), 92-111. http://dx.doi.org/10.1108/14626001311298448.

Mitchelmore, S., y Rowley, J. (2013). Entrepreneurial competencies of women entrepreneurs pursuing business growth. Journal of Small Business and Enterprise Development, 20(1), 125-142. http://dx.doi.org/10.1108/14626001311298448.

Minello, I., Alves L. y Da Costa, L. (2014). Entrepreneurial competencies and business failure. International Journal of Entrepreneurship, 18(1), 1-15 [consultado 6 Ago 2018]. Disponible en: https://www.abacademies.org/articles/ijevol18no12014.pdf.

Moriano, J. Gorgievski, M., Laguna, M., Stephan, U. y Zarafshani, K. (2012). A cross-cultural approach to understanding entrepreneurial intention. Journal of career development, 39(2), 162-185. http://dx.doi.org/10.1177\%2F0894845310384481.

Moska, D., Naranjo, E. y Aguirre, J. (2015). GEM. Reporte Nacional 2014 [consultado 12 Jun 2018]. Disponible en: https://www.gemconsortium.org/economy-profiles/mexico.

Murali, S., Mohani, A. y Yuzliani, Y. (2009). Impact of personal qualities and management skill of entrepreneurs on venture performance in Malaysia: Opportunity recognition skills as a mediating factor. Techno-innovation, 29(11), 798-805. http://dx.doi.org/10.1016/j.technovation.2009.04.002.

Nunnally, J. y Bernstein, I. (1994). Psychometric theory. (3rd Ed.). New York: McGrawHill. Olmos, R., González, R. y Alcaraz, E. (2014). Desarrollo de competencias emprendedoras: Un análisis explicativo con estudiantes universitarios. Cuadernos de Estudios Empresariales, 22(0), 29-53. http://dx.doi.org/10.5209/rev_CESE.2012.v22.44644.

Pablo, I., Santos, B. y Bueno, Y. (2004). Las dimensiones del perfil emprendedor. Contraste empírico con emprendedores de éxito. In: Piqueras UVD (Ed). El emprendedor innovador y la creación de empresas de $I+D+I$, Valencia, España, 813-830 [consultado $10 \mathrm{Jul}$ 2018]. Disponible en: https://www.uv.es/motiva/libromotiva/51PabloSantosBueno.pdf.

Pajarinen, M., Rouvinen, P. y Ylä-Anttila, P. (2006). Growth-orientation of new entrepreneurs in Finland.ETLA, Discussion Papers 1052, 1-77 [consultado 18 Sep 2018]. Disponible en: https://www.researchgate.net/publication/24123239_Uusyrittajien_kasvuhakuisuus.

Partida, A., Carrera, M. y Villarreal, M. (2012). Análisis de las motivaciones, factores de éxito y obstáculos al espíritu empresarial: estudio comparativo entre México, EEUU y Turquía. Innovaciones de Negocios, 9(18), 207-231 [consultado 9 Ago 2018]. Disponible en: http://eprints.uanl.mx/12572/1/A2.pdf.

Quevedo, L., Izar, J. y Romo, L. (2010). Factores endógenos y exógenos de mujeres y hombres emprendedores de España, Estados Unidos y México. Investigación y Ciencia, 46, 57-63 [consultado 15 Ago 2018]. Disponible en: http://redalyc.uaemex.mx/src/inicio/ ArtPdfRed. jsp?iCve=67413508008.

Rehak, J. Bueckmann, R., Barrera, A., Galicia, P. y Haro, J. (2016). Global Entrepreneurship Monitor Queretaro Regional Report 2015, 1-52 [consultado 10 Jul 2018]. Disponible en: http://dx.doi.org/10.13140/RG.2.2.17569.40806. 
Reynolds, P. (1991). Sociology and entrepreneurship. Concepts and contributions. Entrepreneurship Theory and Practice, 16(2), 47-70. http://dx.doi. org/10.1177/104225879201600205.

Sarwoko, E., Surachman, A. y Hadiwidjojo, D. (2013). Entrepreneurial characteristics and competency as determinants of business performance in SMEs. International Organization of Scientific Research. Journal of Business and Management, 7(3), 31-38. http:// dx.doi.org/10.9790/487X-0733138.

Secretaría de Economía, (2014). Informe anual [consultado 17 Jul 2018]. Disponible en: https://www.gob.mx/se/.

Secretaría de Desarrollo Sustentable, (2018). Anuario Estadístico [consultado 24 Jul 2018]. Disponible en: https://www.queretaro.gob.mx.

Stefanovic, I, Prokic, S. y Rankovic, L. (2010). Motivational and success factors of entrepreneurs: the evidence from a developing country. Zb. rad. Ekon. Fak. Rij, 28(2), 251-269 [consultado 28 Jun 2018]. Disponible en https://papers.ssrn.com/sol3/papers. cfm?abstract_id=2267309.

Swierczek, F. y Hathai, T. (2011). Motivation, entrepreneurship and the performance of SMES in Vietnam. Journal of Enterprise Culture, 11(1), 47-68. http://dx.doi.org/10.1142/ S0218495803000044.

Toca, C. (2010). Consideraciones para la formación en emprendimiento. Explorando nuevos ámbitos y posibilidades. Estudios Gerenciales, 26(117), 41-60. http://dx.doi.org/10.1016/ S0123-5923(10)70133-9.

Ucbasaran, D., Westhead, P., Wright, M. y Binks, M. (2003). Does entrepreneurial experience influence opportunity identification? Journal of Private Equity, 7(1), 7-14. http:// dx.doi.org/10.3905/jpe.2003.320059.

Ventura, R. y Quero, M. (2013). Factores explicativos de la intención de emprender en la mujer. Aspectos diferenciales en la población universitaria según la variable género. Cuadernos de Gestión, 13(1), 127-149. http://dx.doi.org/10.5295/cdg.100271rv.

Venkatraman, N. y Ramanujam, V. (1986). Measurement of Business Performance in Strategy Research: A Comparison of Approaches. Academy of Management Review, 11(4), 801-814. http://dx.doi.org/10.2307/258398.

Wennekers, S., Wennekers, A., Thurik, R. y Reynolds, P. (2005). Nascent Entrepreneurship and the Level of Economic Development. Small Business Economic, 24(3), 293-309. http://dx.doi.org/10.1007/s11187-005-1994-8.

Yalcin, S. y Kapu, H. (2008). Entrepreneurial Dimensions in Transitional Economies: A Review of Relevant Literature and the Case of Kyrgyzstan. Journal of Developmental Entrepreneurship, 13(2), 185-204. http://dx.doi.org/10.1142/S1084946708000922.

Zimmerman, M. y Chu, H. (2013). Motivation, Success, and Problems of Entrepreneurs in Venezuela. Journal of Management Policy and Practice, 14(2), 76-90 [consultado 11 Jul 2018]. Disponible en: https://pdfs.semanticscholar.org/e00c/06bcca37e04394d26f1ee9f4 ec6d34b11c84.pdf. 